\title{
Research progress on KIF3B and related diseases
}

\author{
Lihui Zhou, Lian Ouyang, Keying Chen, Xucan Wang \\ Department of Orthopaedic Surgery, Xiangshan First People's Hospital, Ningbo 315700, China \\ Contributions: (I) Conception and design: L Zhou; (II) Administrative support: X Wang; (III) Provision of study materials or patients: K Chen; (IV) \\ Collection and assembly of data: None; (V) Data analysis and interpretation: None; (VI) Manuscript writing: All authors; (VII) Final approval of \\ manuscript: All authors. \\ Correspondence to: Lihui Zhou. Department of Orthopaedic Surgery, Xiangshan First People’s Hospital, Ningbo 315700, China. Email: lihui_chow@163.com.
}

\begin{abstract}
Kinesins constitute a protein superfamily that belongs to the motor protein group. Kinesins move along microtubules to exert their various functions, which include intracellular transportation, mitosis, and cell formation. Kinesins are responsible for the transport of various membrane organelles, protein complexes, mRNA and other material, as well as the regulation of intracellular molecular signal pathways. Cumulative studies have also indicated that kinesins are related to the development of a variety of human diseases. At present, there are 14 subfamilies of the kinesin superfamily (KIFs), comprising 45 members. $\mathrm{KIF} 3$ is the most common expression in KIFs. KIF3 is a complex composed of a KIF3A/3B heterodimer and a kinesin-related protein, known as KAP3. These complexes are organelles and protein complexes involved in membrane binding in various tissues and transport within cells (nerve cells, melanocytes, epithelial cells, etc.). As a member of the KIF3 subfamily, KIF3B is an essential protein that can regulate cell migration, and proliferation and has critical biological functions. During mitosis, KIF3B is responsible for vesicle transport and membrane expansion, thus regulating cell migration. In recent years, more and more attention has been paid to the relationship between KIF3B and the occurrence and development of diseases. This article reviews the recent advances in the study of KIF3B and its related diseases.
\end{abstract}

Keywords: Kinesin superfamily; KIF3B; diseases

Submitted Jul 05, 2019. Accepted for publication Aug 01, 2019.

doi: $10.21037 /$ atm.2019.08.47

View this article at: http://dx.doi.org/10.21037/atm.2019.08.47

\section{Background}

The kinesin superfamily (KIFs) is a class of molecular motors responsible for transporting different molecular cargo along microtubules to their positive poles. It is one of the essential molecules for intracellular transport of cargo such as vesicles, organelles, protein complexes, and RNA. In 1985, Hirokawa et al. first (1) observed linking structures between microtubules and cells using high precision microscopy (rapid-freezing deep-etching). These links were later confirmed to be molecular motor proteins responsible for intracellular transport and included kinesin, dynein, and myosin. Together with other molecular motors, kinesin acts as a transport vehicle at the cellular level to transport different molecules to the designated intracellular location, relying on cytoskeleton structures such as microtubules (2).
There are 14 subfamilies of the KIFs, comprising 45 members. KIF3 is the most common expression in KIFs KIF3B is a member of the KIF3 subfamily, which is an important protein that can regulate cell migration and proliferation and has important biological functions. During mitosis, KIF3B is responsible for vesicle transport and membrane expansion, thus regulating cell migration.

In recent years, more and more attention has been paid to the relationship between KIF3B and the occurrence and development of diseases. This article reviews the recent advances in the study of KIF3B and its related diseases.

\section{Structure, classification, and function of the kinesin superfamily}

KIFs have three domains: motor domains, forkhead- 
associated (FHA) domains, and pleckstrin homology (PH) domains. The motor domains are highly conserved in KIFs, which can combine with ATP and microtubules to provide energy for hydrolysis. Without this domain, the transport capacity for cargo is significantly reduced. The function of the FHA domain is unknown. The $\mathrm{PH}$ domain participates in the transport of cargo and determines the specificity of the combined cargo. KIFs and the intracellular substance transport in which they participate play a vital role in maintaining the essential function of cells.

Since its first discovery in 1985, 45 members from 14 subfamilies of KIF have been identified. The three main types of KIFs have been identified according to their location: amino-terminal motor domain type (N-type), intermediate motor domain type (M-type), and carboxylterminal domain type (C-type) (3). N-type KIFs include KIF1, KIF3, KIF4, KIF5, KIF10, KIF12, KIF13, KIF14, KIF15, KIF16, KIF17, KIF18, KIF20, KIF23, and KIF26. M-Type KIFs only include KIF2. C-Type KIFs include KIFC1, KIFC2, and KIFC3.

At present, there is much known about the function of kinesins, and it is well-established that KIFs are closely related to the occurrence of neurodegeneration, diabetes, nephropathy, and other diseases (4-6). Also, a large number of studies have shown that kinesins are widely involved in the occurrence of various tumors, and its expression levels are directly related to the development of many tumors (7-9).

\section{Overview of KIF3B}

KIF3 is a member of a subfamily in the kinesin superfamilies II, the most common expression in KIFs. It is a complex composed of a KIF3 A/3 B heterodimer and a kinesinrelated protein, known as KAP3 (10). These complexes are organelles and protein complexes involved in membrane binding in various tissues and transport within cells (such as nerve cells, melanocytes, and epithelial cells, etc.) (11-13).

In recent years, more and more attention has been paid to the relationship between KIF3B and the occurrence and development of diseases. KIF3B consists of a large motor molecule, which uses the energy generated from ATP hydrolysis to transport intracellular cargo along microtubules. KIF3B has been located in mitotic organelles (spindle microtubules and centrosomes) during cell division, and its dominant negative mutants have been observed interfering with normal mitosis.

As a kinesin, KIF3B participates in many physiological responses, including mitosis, meiosis, and the transport of macromolecules. Mitosis is a process of eukaryotic cell division, which is complex and highly mediated. During mitosis, KIF3B plays the role of vesicle transport and membrane expansion $(14,15)$, thus regulating cell migration. Any abnormality during mitosis can lead to gene deletion, chromosome translocation, duplication, cell death, and even canceration (11). The KAP3-KIF3A-KIF3B complex can transport proteins from adenomatous polyposis coli (APC) and accumulate at the end of membrane processes, thus regulating cell migration (11).

KIF3B not only regulates cell migration but also regulates the process of the cell cycle, thus promoting cell proliferation and survival. It has essential biological functions and is closely related to the occurrence and development of many diseases (Table 1).

\section{KIF3B and tumors}

Numerous studies have shown that cancer is a disease of the cell cycle. The disorder of cell cycle regulation can be a feature of cancer, indicating that the abnormal regulation of the cell cycle may lead to the occurrence of cancer. The relationship between kinesins and tumors has been intensely researched in recent years. Because kinesins participate in many cell processes, including mitosis, meiosis, and the transport of macromolecules, several kinesins have been reported to be associated with cancer, and participate in the proliferation, dedifferentiation, invasion, and metastasis of cancer cells and anti-apoptosis processes (31-34). Kinesins are expressed in cancer tissues and healthy tissues and can be used as a diagnostic marker for many kinds of tumors, although the specific molecular mechanism involved is still unclear. If the roles of kinesins in tumorigenesis, development, invasion, and metastasis can be clarified, it will be of great significance for the early diagnosis and treatment of tumors. At present, there are a few reports on the relationship between KIF3B and the tumorigenesis and development of cancers, including hepatocellular carcinoma, pancreatic cancer, cervical cancer, and other diseases.

\section{Hepatocellular carcinoma (HCC)}

HCC is the fifth most common cancer, and the second most common cause of cancer-related death in the world (35). In clinical studies (16), it has been confirmed that KIF3B levels are higher in HCC tissues when compared with those in para-carcinoma tissues. Also, the expression of KIF3B 
Table 1 KIF3B and related diseases

\begin{tabular}{|c|c|c|c|}
\hline Disease & KIF3B expression & Results & Reference \\
\hline \multirow{3}{*}{$\begin{array}{l}\text { Hepatocellular } \\
\text { carcinoma }\end{array}$} & \multirow{3}{*}{$\begin{array}{l}\text { Highly expressed in } \\
\text { cancer tissues }\end{array}$} & $\begin{array}{l}\text { KIF3B level was significantly associated with the histological grade, } \\
\text { tumor size, and serum AFP level }\end{array}$ & \multirow{3}{*}{$(16,17)$} \\
\hline & & High KIF3B level was significantly associated with poor prognosis & \\
\hline & & $\begin{array}{l}\text { The growth of hepatocellular carcinoma cells decreased significantly } \\
\text { after inhibiting KIF3B }\end{array}$ & \\
\hline Pancreatic cancer & $\begin{array}{l}\text { Highly expressed in } \\
\text { cancer tissues }\end{array}$ & $\begin{array}{l}\text { KIF3B was closely related to TNM staging, lymph node metastasis, } \\
\text { and vascular invasion }\end{array}$ & (18) \\
\hline \multirow[t]{2}{*}{ Spermatogonial cancer } & \multirow[t]{2}{*}{$\begin{array}{l}\text { Highly expressed in } \\
\text { cancer tissues }\end{array}$} & $\begin{array}{l}\text { The knockdown of KIF3B significantly suppressed cell migration and } \\
\text { viability }\end{array}$ & \multirow[t]{2}{*}{$(20)$} \\
\hline & & $\begin{array}{l}\text { The rates of multipolar division and multi-nucleation were raised when } \\
\text { KIF3B was knocked down }\end{array}$ & \\
\hline Cervical cancer & $\begin{array}{l}\text { Highly expressed in } \\
\text { cancer tissues }\end{array}$ & Remains elusive & $(21,22)$ \\
\hline $\begin{array}{l}\text { Polycystic kidney } \\
\text { disease }\end{array}$ & - & $\begin{array}{l}\text { Related to intracellular transport of mesodermal active primary cilia- } \\
\text { forming materials }\end{array}$ & $(23-25)$ \\
\hline Dent's disease & $\begin{array}{l}\text { Expressed in kidney } \\
\text { tissues }\end{array}$ & $\begin{array}{l}\text { Interacted with CLC-5 for CLC- } 5 \text { plasma membrane expression, } \\
\text { endocytosis, and microtubular transport in the kidney }\end{array}$ & $(29)$ \\
\hline \multirow[t]{2}{*}{ Spinal cord injury } & \multirow{2}{*}{$\begin{array}{l}\text { Increased KIF3B } \\
\text { expression after acute } \\
\text { spinal cord injury }\end{array}$} & $\begin{array}{l}\text { The activation and proliferation of microglia and astrocytes after } \\
\text { spinal cord injury }\end{array}$ & \multirow[t]{2}{*}{$(10,30)$} \\
\hline & & $\begin{array}{l}\text { High expression of KIF3B after spinal cord injury was closely related } \\
\text { to functional recovery }\end{array}$ & \\
\hline
\end{tabular}

and $\mathrm{Ki} 67$ in HCC tissues has been positively correlated, suggesting that KIF3B is associated with abnormal proliferation of hepatocellular carcinoma and may be involved in the occurrence and development of HCC. Also, an analysis of the relationship between KIF3B level and clinicopathological factors and prognosis revealed that KIF3B level was significantly associated with the histological grade, tumor size, and serum AFP level. Survival analysis also showed that a high KIF3B level was significantly associated with poor prognosis in HCC patients.

In previous studies (17), it was found that after stimulating cell proliferation with serum starvation and serum release, the expression level of p27kip1 as a negative regulator of cell cycle was down-regulated, while the 
expression levels of KIF3B, cyclinA, and CDK2 were upregulated, indicating that the expression of CyclinA and CDK2 in HepG2 cells changed with the expression of KIF3B. These results suggest that KIF3B can regulate the changes in cyclin A, CDK2, and p27kip1. It was found that the growth of hepatocellular carcinoma cells decreased significantly after inhibiting KIF3B and that the proportion of the G1 phase of cell cycle increased, while the proportion of the $\mathrm{S}$ phase decreased, thus leading to apoptosis. The results showed that the loss of KIF3B expression resulted in the inhibition of cell proliferation and cell cycle arrest in the G1 phase, which suggests that abnormal expression of KIF3B may lead to abnormal regulation of the cell cycle.

Taken together, the results above suggest that KIF3B is involved in the pathogenesis of hepatocellular carcinoma and may be an independent prognostic factor and potential therapeutic target for human HCC.

\section{Pancreatic cancer}

A recent study (18) showed that KIF3B was highly expressed in human pancreatic cancer tissues, and the expression of KIF3B was closely related to pTNM staging, lymph node metastasis, and vascular invasion. Moreover, the high expression of KIF3B predicted poor prognosis in pancreatic cancer patients. Functional assays revealed that knockdown KIF3B in vitro and in vivo inhibited cancer cell proliferation by affecting Ki67 and proliferating cell nuclear antigen (PCNA). These data suggest that KIF3Bis associated with pancreatic cancer malignant progression, especially proliferation. Hence, KIF3B might serve as a potential therapeutic target for the clinical treatment of pancreatic cancer.

\section{Oral squamous cell carcinoma (OSCC)}

A study (19) showed that KIF3B was highly expressed in OSCC, which promoted tumorigenesis of OSCC. Further analysis indicated that KIF3B was a direct target of miR127-3p. The upregulation of KIF3B partially attenuated the inhibitory effect of miR-127-3p on the development of OSCC.

\section{Spermatogonial cancer}

Testis cancer is common cancer among young men, with over $50 \%$ of the cases being seminomas. However, the exact mechanism of this disease remains unclear.
A study (20) showed that the protein levels of KIF3B were enriched in seminoma tissue in comparison to those of healthy testicular tissues, and the KIF3B was mainly expressed in cells with division potential. The knockdown of KIF3B significantly suppressed cell migration and viability. Immunofluorescence images revealed KIF3 B mainly gathered at the spindles and was enriched at the central spindle, which indicates KIF3B may have a direct impact on spindle formation and cytokinesis. It was also found that the rates of multipolar division and multi-nucleation were raised when KIF3B was knocked down. The cumulative results above demonstrate that KIF3B is important in mitosis and may be essential to seminoma cell division and proliferation.

\section{Cervical cancer (CC)}

In CC, a preliminary genomic analysis $(21,22)$ was conducted to identify essential genes that contribute to tumor initiation and progression of CC. In this study, single nucleotide polymorphism (SNP) array, gene expression profiling, and fluorescence in situ hybridization were conducted. A total of 26 overexpressed genes were identified, several which were functionally important genes in cell cycle regulation, including KIF3B. Despite these findings, the specific role and underlying mechanism remain elusive. These studies $(21,22)$ represent an essential step toward the development of clinically significant biomarkers in CC.

\section{KIF3B and nephropathy}

\section{Polycystic kidney disease (PKD)}

PKD is a common hereditary disease characterized by the accumulation of fluid-filled cysts in the kidney, liver, and other organs $(36,37)$. Several proteins encoded by polycystic kidney disease-related genes have been identified in primary cilia. Also, recent observations have shown that primary ciliary abnormalities play a role in cyst formation. Primary cilia are hairy organelles surrounded by nine pairs of peripheral microtubules and cilia. The synthesis of primary cilia is related to the inhibition of intraciliary transport, in which large particles containing transported proteins are transported along the ciliary axis (38). KIF3 mediates protein migration along the axis. In Hirokawa's and Goldstein's experiments, mutant mice encoding KIF3B subunits resulted in a lethal phenotype (23-25). The cilia of nodes are relatively aggregated with the heterotrimeric 
trimer KIF3, and the one-way flow of extraembryonic fluid (node flow) generated by rotation can fundamentally control left-right symmetry (23). In KIF3B-negative mutants, there is no node flow. Therefore, KIF3B is necessary for the use of left-right axisymmetry, mainly because it is related to the intracellular transport of mesodermal active primary ciliaforming materials (23).

\section{Distal renal tubular acidosis}

Duangrum et al. (5) showed that human kidney anion exchanger $1(\mathrm{kAE} 1)$ and KIF3B were co-expressed in human kidney tissues and bound to KIF3B through dileucine motif at the carboxyl end. Inhibition of KIF3B in HEK293T cells inhibited the content of kAE1 on the cell membrane, resulting in the accumulation of $\mathrm{kAE} 1$ in cells. These results suggest that KIF3B may be involved in the accumulation of kAE1 in HEK293T cells. KAE1 is transported to the cell membrane in human renal intercalated cells, resulting in the formation of distal renal tubular acidosis.

\section{Kartagener's syndrome}

In Kartagener's syndrome, the combination of abnormal defects in nodal cilia, ciliary bronchi, and sperm flagellum is also an obstacle to KIF3's intracellular transport. In anterograde transport, KIF3 has been found in many typical neurons, except cilia (26,39-41). In specific subsets of Drosophila nerve cells, the absence of KIF3 mutants indicated a decrease of the transport of soluble choline acetyltransferase from the soma to the synapse (27).

\section{Renal ischemia/reperfusion injury}

Renal ischemia/reperfusion injury (I/R) is the basis of renal transplantation and acute kidney injury. A study showed that KIF3B was closely related to I/R. It was found (28) that miR-127 was induced in the rat proximal tubule cells submitted to I/R mimicking conditions, which further targeted KIF3B to regulate intracellular trafficking. These data indicate that KIF3B may be the critical mediator of proximal epithelial tubule cells in response to I/R.

\section{Dent's disease}

Dent's disease is characterized by low-molecular-weight proteinuria, hypercalciuria, nephrolithiasis, and renal failure, which is due to the mutations in CLC-5. CLC-5 is expressed in endosomes and apical membranes of renal tubules. CLC-5 is responsible for receptor-mediated endocytosis and the trafficking of megalin and cubilin. It was reported that the $\mathrm{COOH}$ terminus of CLC-5 interacted with the coiled-coil and globular domains of KIF3B to facilitate fast anterograde translocation of membranous organelles.

Furthermore, it was demonstrated that CLC-5 was associated with with KIF3B, resulting in the transport of CLC-5-containing vesicles along KIF3B microtubules. The effects of overexpressed KIF3B on albumin endocytosis were dependent on CLC-5 expression. These findings indicate that the interaction of CLC-5 and KIF3B is essential for CLC-5 plasma membrane expression, endocytosis. Also, microtubular transport in the kidney (29).

\section{KIF3B and spinal cord injury}

Spinal cord injury refers to acute traumatic insults to the neural components in the spinal canal (42). Previous studies $(10,30)$ have shown that the expression of KIF3B increased significantly after acute spinal cord injury in adult rats, mainly in microglia and astrocytes, and that the high expression of KIF3B is closely related to the activation and proliferation of microglia and astrocytes after spinal cord injury. Recent studies have shown that IL- $1 \beta$ overexpression in adult rats with acute spinal cord injury can delay the functional recovery of spinal cord injury by inducing the down-regulation of KIF3B expression by miR-372. This suggests that the high expression of KIF3B after spinal cord injury is closely related to functional recovery.

\section{Prospect}

Kinesin is a motor protein which was isolated from the axon plasm of squid in 1985. Among 14 subfamilies, 45 members of KIFs, KIF3 is the most common expression in KIFs., and KIF3B is an essential member of the KIF3 subfamily. Many studies have revealed their different functions. However, the relationship between KIF3B and disease still poses many intriguing but as yet unanswered questions. Understanding the interaction between KIF3B and transport will be critical for unveiling the complexity of human diseases, and further clarifying the regulatory mechanism. The extensive expression of KIF3B and the existing evidence prove it to be effective in maintaining the normal life activities of cells, and the abnormal expression may lead to many diseases. Therefore, further research on KIF3B has significant 
clinical significance in the future.

\section{Acknowledgments}

Funding: This work was supported by the Project of Medical Technology of Zhejiang province (No. 2017KY611).

\section{Footnote}

Conflicts of Interest: The authors have no conflicts of interest to declare.

Ethical Statement: The authors are accountable for all aspects of the work in ensuring that questions related to the accuracy or integrity of any part of the work are appropriately investigated and resolved.

\section{References}

1. Hirokawa N, Bloom GS, Vallee RB. Cytoskeletal architecture and immunocytochemical localization of microtubule-associated proteins in regions of axons associated with rapid axonal transport: the beta, beta'iminodipropionitrile-intoxicated axon as a model system. J Cell Biol 1985;101:227-39.

2. Hirokawa N, Niwa S, Tanaka Y. Molecular motors in neurons: transport mechanisms and roles in brain function, development, and disease. Neuron 2010;68:610-38.

3. Hirokawa N. Kinesin and dynein superfamily proteins and the mechanism of organelle transport. Science 1998;279:519-26.

4. Ari C, Borysov SI, Wu J, et al. Alzheimer amyloid beta inhibition of Eg5/kinesin 5 reduces neurotrophin and/ or transmitter receptor function. Neurobiol Aging 2014;35:1839-49.

5. Duangtum N, Junking $M$, Sawasdee $N$, et al. Human kidney anion exchanger 1 interacts with kinesin family member 3B (KIF3B). Biochem Biophys Res Commun 2011;413:69-74.

6. Lockerbie RO, Edde B, Prochiantz A. Cyclic AMPdependent protein phosphorylation in isolated neuronal growth cones from developing rat forebrain. J Neurochem 1989;52:786-96.

7. Chandrasekaran G, Tatrai P, Gergely F. Hitting the brakes: targeting microtubule motors in cancer. Br J Cancer 2015;113:693-8.

8. Chen S, Stout JR, Dharmaiah S, et al. Transient endoreplication down-regulates the kinesin-14 HSET and contributes to genomic instability. Mol Biol Cell 2016;27:2911-23.

9. Kato T, Lee D, Wu L, et al. Kinesin family members KIF11 and KIF23 as potential therapeutic targets in malignant pleural mesothelioma. Int J Oncol 2016;49:448-56.

10. Yu X, Wen H, Cao J, et al. Temporal and spatial expression of KIF3B after acute spinal cord injury in adult rats. J Mol Neurosci 2013;49:387-94.

11. Jimbo T, Kawasaki Y, Koyama R, et al. Identification of a link between the tumour suppressor APC and the kinesin superfamily. Nat Cell Biol 2002;4:323-7.

12. Tuma MC, Zill A, Le Bot N, et al. Heterotrimeric kinesin II is the microtubule motor protein responsible for pigment dispersion in Xenopus melanophores. J Cell Biol 1998; $143: 1547-58$

13. Takeda S, Yamazaki H, Seog DH, et al. Kinesin superfamily protein 3 (KIF3) motor transports fodrinassociating vesicles important for neurite building. J Cell Biol 2000;148:1255-65.

14. Fan J, Beck KA. A role for the spectrin superfamily member Syne-1 and kinesin II in cytokinesis. J Cell Sci 2004;117:619-29.

15. Keil R, Kiessling C, Hatzfeld M. Targeting of p0071 to the midbody depends on KIF3. J Cell Sci 2009;122:1174-83.

16. Sun $X$, Jin Z, Song $X$, et al. Evaluation of KIF23 variant 1 expression and relevance as a novel prognostic factor in patients with hepatocellular carcinoma. BMC Cancer 2015; 15:961.

17. Huang X, Liu F, Zhu C, et al. Suppression of KIF3B expression inhibits human hepatocellular carcinoma proliferation. Dig Dis Sci 2014;59:795-806.

18. Liu ZH, Dong SX, Jia JH, et al. KIF3B Promotes the Proliferation of Pancreatic Cancer. Cancer Biother Radiopharm 2019;34:355-61.

19. Ji L, Zhu ZN, He CJ, et al. MiR-127-3p targets KIF3B to inhibit the development of oral squamous cell carcinoma. Eur Rev Med Pharmacol Sci 2019;23:630-40.

20. Shen HQ, Xiao YX, She ZY, et al. A novel role of KIF3b in the seminoma cell cycle. Exp Cell Res 2017;352:95-103.

21. Narayan G, Murty VV. Integrative genomic approaches in cervical cancer: implications for molecular pathogenesis. Future Oncol 2010;6:1643-52.

22. Scotto L, Narayan G, Nandula SV, et al. Identification of copy number gain and overexpressed genes on chromosome arm 20q by an integrative genomic approach in cervical cancer: potential role in progression. Genes Chromosomes Cancer 2008;47:755-65. 
23. Nonaka S, Tanaka Y, Okada Y, et al. Randomization of left-right asymmetry due to loss of nodal cilia generating leftward flow of extraembryonic fluid in mice lacking KIF3B motor protein. Cell 1998;95:829-37.

24. Takeda S, Yonekawa Y, Tanaka Y, et al. Left-right asymmetry and kinesin superfamily protein KIF3A: new insights in determination of laterality and mesoderm induction by kif3A-/- mice analysis. J Cell Biol 1999;145:825-36.

25. Marszalek JR, Ruiz-Lozano P, Roberts E, et al. Situs inversus and embryonic ciliary morphogenesis defects in mouse mutants lacking the KIF3A subunit of kinesin-II. Proc Natl Acad Sci U S A 1999;96:5043-8.

26. Yamazaki H, Nakata T, Okada Y, et al. KIF3A/B: a heterodimeric kinesin superfamily protein that works as a microtubule plus end-directed motor for membrane organelle transport. J Cell Biol 1995;130:1387-99.

27. Ray K, Perez SE, Yang Z, et al. Kinesin-II is required for axonal transport of choline acetyltransferase in Drosophila. J Cell Biol 1999;147:507-18.

28. Aguado-Fraile E, Ramos E, Saenz-Morales D, et al. miR-127 protects proximal tubule cells against ischemia/ reperfusion: identification of kinesin family member $3 \mathrm{~B}$ as miR-127 target. PLoS One 2012;7:e44305.

29. Reed AA, Loh NY, Terryn S, et al. CLC-5 and KIF3B interact to facilitate CLC-5 plasma membrane expression, endocytosis, and microtubular transport: relevance to pathophysiology of Dent's disease. Am J Physiol Renal Physiol 2010;298:F365-80.

30. Zhou W, Yuan T, Gao Y, et al. IL-1beta-induces NFkappaB and upregulates microRNA-372 to inhibit spinal cord injury recovery. J Neurophysiol 2017;117:2282-91.

31. Taniuchi K, Nakagawa H, Nakamura T, et al. Downregulation of RAB6KIFL/KIF20A, a kinesin involved with membrane trafficking of discs large homologue 5, can attenuate growth of pancreatic cancer cell. Cancer Res

Cite this article as: Zhou L, Ouyang L, Chen K, Wang X. Research progress on KIF3B and related diseases. Ann Transl Med 2019;7(18):492. doi: 10.21037/atm.2019.08.47
2005;65:105-12.

32. Corson TW, Gallie BL. KIF14 mRNA expression is a predictor of grade and outcome in breast cancer. Int J Cancer 2006;119:1088-94.

33. Nakamura Y, Tanaka F, Haraguchi N, et al. Clinicopathological and biological significance of mitotic centromere-associated kinesin overexpression in human gastric cancer. Br J Cancer 2007;97:543-9.

34. Taniwaki M, Takano A, Ishikawa N, et al. Activation of KIF4A as a prognostic biomarker and therapeutic target for lung cancer. Clin Cancer Res 2007;13:6624-31.

35. Torre LA, Bray F, Siegel RL, et al. Global cancer statistics, 2012. CA Cancer J Clin 2015;65:87-108.

36. Igarashi P, Somlo S. Genetics and pathogenesis of polycystic kidney disease. J Am Soc Nephrol 2002;13:2384-98.

37. Lin F, Hiesberger T, Cordes K, et al. Kidney-specific inactivation of the KIF3A subunit of kinesin-II inhibits renal ciliogenesis and produces polycystic kidney disease. Proc Natl Acad Sci U S A 2003;100:5286-91.

38. Rosenbaum JL, Witman GB. Intraflagellar transport. Nat Rev Mol Cell Biol 2002;3:813-25.

39. Kondo S, Sato-Yoshitake R, Noda Y, et al. KIF3A is a new microtubule-based anterograde motor in the nerve axon. J Cell Biol 1994;125:1095-107.

40. Muresan V, Abramson T, Lyass A, et al. KIF3C and KIF3A form a novel neuronal heteromeric kinesin that associates with membrane vesicles. Mol Biol Cell 1998;9:637-52.

41. Yang Z, Goldstein LS. Characterization of the KIF3C neural kinesin-like motor from mouse. Mol Biol Cell 1998;9:249-61.

42. Zhou L, Ouyang L, Lin S, et al. Protective role of betacarotene against oxidative stress and neuroinflammation in a rat model of spinal cord injury. Int Immunopharmacol 2018;61:92-9. 